\title{
CUIDADO EM SAÚDE DAS MULHERES GRÁVIDAS PRIVADAS DE LIBERDADE: REVISÃO INTEGRATIVA
}

\author{
HEALTH CARE OF PREGNANT WOMEN DEPRIVED \\ OF THEIR LIBERTY: INTEGRATIVE REVIEW
}

\section{ATENCIÓN MÉDICA PARA LAS MUJERES EMBARAZADAS PRIVADAS DE LIBERTAD: REVISIÓN INTEGRADORA}

\author{
Ana Carolina Sales ${ }^{1}$ \\ Gabrielle Kimie Pinheiro Nakada ${ }^{2}$ \\ Mateus Rodrigo Palombit ${ }^{3}$ \\ Vander Monteiro Conceição ${ }^{4}$ \\ Sueli Santiago Baldan \\ Elaine Miguel Delvivo Farão \\ Rafaela Azevedo Abrantes de Oliveira Simoneti ${ }^{7}$
}

\begin{abstract}
Como citar esse artigo: Sales AC, Nakada GKP, Palombit MR, Conceição VM, Bandan SS, Farão EMD, et al. Cuidado em saúde das mulheres grávidas privadas de liberdade: revisão integrativa. Rev baiana enferm. 2021;35:e36114.

Objetivo: analisar as publicações nacionais e internacionais acerca da assistência ao pré-natal oferecida às mulheres privadas de liberdade. Método: revisão integrativa da literatura. As buscas foram realizadas em bases de dados, usando descritores e palavras-chave separadas por operadores boleanos. A busca resultou em 548 estudos. Após análise dos critérios de inclusão e exclusão com leitura analítica, foram selecionados 11 estudos primários, que foram organizados nas categorias estudos nacionais e internacionais. Resultados: são necessárias adequações e melhorias na assistência à saúde dessas mulheres que, mesmo tendo direitos vigentes, sofrem negligência na assistência oferecida. Conclusão: a vivência do pré-natal no cárcere está repleta de dificuldades e peculiaridades a depender da penitenciária, exigindo dos gestores e dos profissionais de saúde um olhar atento às necessidades da mulher, da criança e de seus familiares, na tentativa de minimizar os impactos do contexto prisional na qualidade de vida das pessoas envolvidas.
\end{abstract}

Descritores: Prisões. Gravidez. Mulheres. Enfermagem.

Objective: to analyze national and international publications on prenatal care offered to women deprived of their liberty. Method: integrative literature review. Searches were conducted in databases, using descriptors and keywords separated by Boolean operators. The search resulted in 548 studies. After analysis of inclusion and exclusion criteria

\footnotetext{
Enfermeira. Pesquisadora independente. Coxim, Mato Grosso do Sul, Brasil. https://orcid.org/0000-000 I-7555-7098.

Enfermeira. Pesquisadora independente. Coxim, Mato Grosso do Sul, Brasil. https://orcid.org/0000-0002-9687-5500.

Estudante de Enfermagem. Universidade Federal da Fronteira Sul. Chapecó, Santa Catarina, Brasil. https://orcid.org/0000-0003-3199-6384.

Enfermeiro. Doutor em Enfermagem Fundamental. Docente da Universidade Federal da Fronteira Sul. Chapecó, Santa Catarina, Brasil. https://orcid.org/0000-00030972-0795.

Enfermeira. Doutora em Promoção de Saúde. Docente da Universidade Federal de Mato Grosso do Sul. Coxim, Mato Grosso do Sul, Brasil. https://orcid.org/00000002-2823-0057.

Enfermeira. Mestre em Enfermagem. Docente da Universidade Federal de Mato Grosso do Sul. Coxim, Mato Grosso do Sul, Brasil. http://orcid.org/0000-00028089-2197.

Enfermeira. Doutora em Enfermagem Fundamental. Docente da Universidade Federal de Pernambuco. Vitória de Santo Antão, Pernambuco, Brasil. rafaelazevedo84@gmail.com. https://orcid.org/0000-0002-4694-2197.
} 
with analytical reading, 11 primary studies were selected, which were organized in the categories national and international studies. Results: adjustments and improvements in health care are needed for these women who, even though they have existing rights, are negligent in the care offered. Conclusion: the prenatal experience in prison is full of difficulties and peculiarities depending on the penitentiary, requiring managers and health professionals an attentive look at the needs of women, children and their family members, in an attempt to minimize the impacts of the prison context on the quality of life of the people involved.

Descriptors: Prisons. Pregnancy. Women. Nursing.

Objetivo: analizar las publicaciones nacionales e internacionales sobre la atención prenatal ofrecida a las mujeres privadas de libertad. Método: revisión integradora de la literatura. Las búsquedas se realizaron en bases de datos, utilizando descriptores y palabras clave separadas por operadores boleanos. La búsqueda dio como resultado 548 estudios. Tras analizar los criterios de inclusión y exclusión con lectura analítica, se seleccionaron 11 estudios primarios, que se organizaron en las categorias de estudios nacionales e internacionales. Resultados: se necesitan ajustes y mejoras en el cuidado de la salud de estas mujeres que, a pesar de tener derechos existentes, son negligentes en el cuidado ofrecido. Conclusión: la experiencia prenatal en la cárcel está llena de dificultades y peculiaridades según el centro penitenciario, lo que exige que los gestores y los profesionales de la salud examinen detenidamente las necesidades de las mujeres, los niños y sus familias, en un intento por reducir al mínimo los efectos del contexto carcelario en la calidad de vida de las personas implicadas.

Descriptores: Cárceles. Embarazo. Las mujeres. Enfermería.

\section{Introdução}

O aumento da população carcerária feminina é expressivo. Mais de 714.000 mulheres estão presas ao redor do mundo. Elas representam 7\% da população carcerária global. Nos países africanos, a proporção de mulheres na população carcerária é de 3\%, sendo muito menor do que em outros lugares. Nas Américas, mulheres representam 8\% do total da população prisional (6\%, se os valores dos Estados Unidos da América forem excluídos). Na Ásia 7\%, na Europa 6\% (5\% se for excluída a Rússia), e na Oceania 7\%. O país com as maiores proporções de prisioneiras é Hong Kong, na China (21\%) ${ }^{(1)}$. O Brasil destaca-se na evolução da taxa de aprisionamento do gênero feminino, com aumento de 455\% entre os anos de 2000 e 2016. Em junho de 2016, no Brasil, o número de Mulheres Privadas de Liberdade (MPL) chegou ao total de $42.355^{(2)}$. Este número passou por um considerável aumento, pois, em 2014, o número de presidiárias era de $37.380^{(3)}$.

Analisando esse contexto, têm-se altos índices de MPL, bem como cadeias que enaltecem uma realidade repulsiva para a sociedade, que traz a mulher fugindo da premissa de "sexo frágil", para aquela que gera e pratica violência, contrabando ou demais delitos ${ }^{(4)}$. Em contrapartida, uma vez presa, o processo de reeducação deve ser garantido e não estrangulado pela incapacidade de ressocialização ou falta de manutenção de princípios, como promoção e manutenção da saúde dessas mulheres. No entanto, o que ainda opera é um ambiente de enjaulamento "adoecedor" tanto físico como mental ${ }^{(5)}$.

Paralelamente, destaca-se aqui o contexto de que, independentemente do tempo que a mulher passar no cárcere, não se pode negligenciar que ela é ou será mãe durante o aprisionamento. Daí a relevância deste estudo. Há uma lacuna que precisa ser explorada dentro desse cenário. Como é desenvolvido o cuidado em saúde durante o pré-natal de mulheres privadas de liberdade?

No que tange ao contexto de saúde dessas mulheres, tem-se, no Brasil, o Plano Nacional de Saúde no Sistema Penitenciário (PNSSP), que prevê a inclusão da população carcerária no Sistema Único de Saúde (SUS), contribuindo para a promoção da saúde e para o controle e/ou redução dos agravos mais frequentes que as acometem. O PNSSP possui como linha de ação direcionada à saúde da mulher a realização de 
pré-natal e o controle do câncer cérvico-uterino e de mama, com o objetivo de implantar, em 100\% das unidades penitenciárias, ações educativas sobre pré-natal, parto, puerpério e anticoncepção, dentre outros temas ${ }^{(6)}$.

Contrário ao descrito na PNSSP, prevalece, nas penitenciárias, dificuldades em manter condições dignas de qualidade de vida àquelas privadas de liberdade. São acomodações improvisadas, escassez de profissionais de saúde, muitas delas carregam o estigma de um ambiente de alta periculosidade, bem como falta de recursos e medicamentos necessários à assistência à saúde. As práticas de prevenção e promoção da saúde e programas como o pré-natal são praticamente inexistentes nesse cenário. Logo, há um paradoxo entre o que é sentido e vivido na prática e o que é referido nas políticas públicas do Brasil ${ }^{(5)}$. Vivencia-se um contexto em que o número de mulheres privadas de liberdade só aumenta, mas as estruturas carcerárias mantêm-se as mesmas ou são ainda mais precárias e negligenciadas.

No sistema prisional, a equipe de enfermagem pode atuar em conjunto com uma equipe multidisciplinar, a fim de prestar uma assistência adequada às MPL na realização de consultas de enfermagem, consultas de pré-natal, exames citopatológicos e de mamas ${ }^{(7)}$. O enfermeiro exerce função fundamental no serviço de saúde. Sua atuação envolve desde promoção e prevenção da saúde até processo administrativo e questões agudas e crônicas do cuidar. Entretanto, é necessário garantir condições sustentáveis de trabalho, o que ainda é um desafio.

Com o crescimento carcerário feminino e a necessidade de manutenção de qualidade de vida e assistência à saúde, aqui focado no período do pré-natal, justifica-se a necessidade do presente estudo, por detalhar e avaliar como o cuidado em saúde está sendo ofertado a essas MPL. Portanto, o objetivo deste estudo é analisar as publicações nacionais e internacionais acerca da assistência ao pré-natal oferecida às mulheres privadas de liberdade.

\section{Método}

A revisão integrativa (RI) é um método utilizado para analisar e sintetizar as pesquisas, contribuindo para o aprofundamento da temática, bem como para síntese de conhecimento por meio de um processo sistemático e rigoroso ${ }^{(8)}$.

A condução da revisão deve seguir os mesmos princípios de rigor metodológico de uma pesquisa. Para tanto, devem ser seguidas as seguintes etapas: identificação do tema e seleção da questão da pesquisa; estabelecimento de critérios para inclusão e exclusão de estudos (seleção de amostra); extração dos dados dos estudos primários; avaliação crítica dos estudos incluídos; síntese dos resultados da revisão; e apresentação da revisão ${ }^{(8-9)}$.

A realização da revisão integrativa foi amparada também pelo modelo Preferred Reporting Items for Systematic Reviews and Meta-Analyses

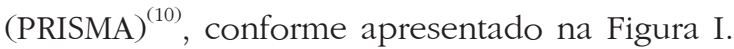
A elaboração da questão norteadora foi baseada na estratégia PICO, que consiste na identificação do $\mathrm{P}=$ participante, $\mathrm{I}=$ intervenção, $\mathrm{C}=$ controle e $\mathrm{O}=$ desfecho, e abrangeu a população (mulheres grávidas encarceradas), o interesse do estudo (identificar a assistência à saúde desse grupo), o contexto (cuidado em saúde das mulheres grávidas privadas de liberdade) e o desfecho (qualidade do cuidado prestado a esse grupo). 
Figura 1 - Adaptação do Flow Diagrama do processo de seleção de artigos da revisão integrativa, de acordo com o PRISMA

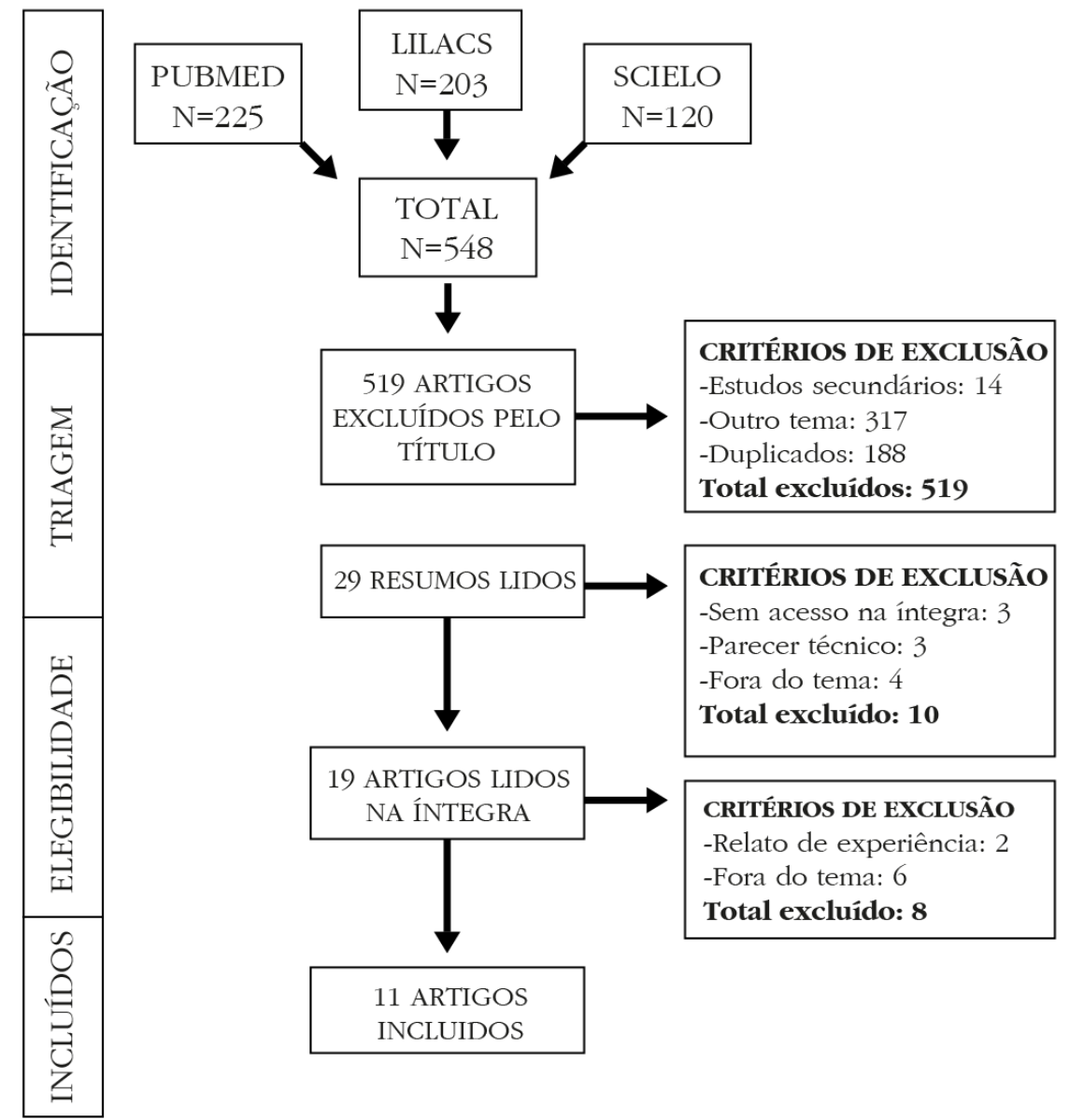

Fonte: Elaboração própria.

A questão norteadora da presente revisão integrativa foi: O que as publicações abordam acerca da assistência ao pré-natal no sistema prisional?

Para a busca dos estudos primários, utilizou-se o portal de periódico online Scientific Eletronic Library Online (SciELO), a base de dados Centro Latino-Americano e do Caribe de Informação em Ciências da Saúde (LILACS) e National Library of Medicine (PubMed).

A busca foi realizada em abril de 2019, concomitantemente, nas bases de dados, com utilização de descritores controlados (vocabulário específico de cada base de dados). Deste modo, nos portais de busca SciELO e LILACS foram utilizados os seguintes descritores controlados: Saúde da mulher $A N D$ prisões $A N D$ gravidez/Gestantes $A N D$ prisões/Mulheres $A N D$ prisões/Mulheres $A N D$ Prisões. Na base de dados
PubMed foram utilizados os seguintes descritores controlados: Pregnancy AND Prision AND bealth care/Prision AND Pregnancy/Pregnancy AND Prision. Tais descritores foram combinados, utilizando-se o operador boleano $A N D$ até que se obtivessem os estudos correspondentes aos critérios de inclusão e exclusão delimitados.

Os critérios de inclusão estabelecidos foram: estudos primários - devido ao elevado número de estudos identificados na busca nos portais de pesquisa, delimitou-se que seriam selecionados artigos a partir de 2009, disponíveis na íntegra, em português e inglês; trabalhos que envolvessem os cuidados em saúde acerca da mulher grávida privada de liberdade; trabalhos que envolvessem mulheres grávidas independente da semana da gestação; estudos que abordassem os cuidados de saúde durante a gestação e o parto. Os critérios de exclusão adotados foram: 
estudos que abordassem especificamente o filho das mulheres privadas de liberdade; estudos que abordassem especificamente o puerpério.

O nível de evidência faz parte da etapa de avaliação crítica dos estudos incluídos na revisão ${ }^{(8)}$ e auxiliam os autores a delinear o perfil metodológico dos estudos, além de permitir avaliar a credibilidade científica das pesquisas. Os estudos primários selecionados foram qualificados pelo nível de evidência ${ }^{(11)}$. No nível I, as evidências são provenientes de revisão sistemática ou meta-análise de todos os relevantes ensaios clínicos randomizados controlados ou oriundos de diretrizes clínicas baseadas em revisões sistemáticas de ensaios clínicos randomizados controlados; nível II, evidências derivadas de pelo menos um ensaio clínico randomizado controlado bem delineado; nível III, evidências obtidas de ensaios clínicos bem delineados sem randomização; nível IV, evidências provenientes de estudo de coorte e de caso controle bem delineados; nível $\mathrm{V}$, evidências originárias de revisão sistemática de estudos descritivos e qualitativos; nível VI, evidências derivadas de um único estudo descritivo ou qualitativo; nível VII, evidências oriundas de opinião de autoridades e/ou relatórios de comitês de especialistas ${ }^{(11)}$.

A análise descritiva foi adotada para a organização e discussão dos resultados evidenciados, na qual foi apresentada uma síntese de cada estudo incluído na revisão, organizados em categorias temáticas. Os artigos, quando citados nas tabelas, são representados pela simbologia alfanumérica E1, E2... E11.

\section{Resultados}

Foram identificados, preliminarmente, 548 registros por meio da busca nos portais LILACS, SciELO e PubMed. Após leitura do título, foram eliminados 188 artigos que apareceram mais de uma vez nas buscas realizadas e artigos publicados há mais de 10 anos, e 317 artigos que não estavam em consonância com o tema proposto. Após essa etapa, foi realizada a leitura dos resumos dos artigos. De acordo com os critérios de inclusão e exclusão descritos na Figura 1, chegou-se ao número de 11 estudos que compuseram a presente revisão integrativa.

Com relação à caracterização dos estudos primários, oito foram desenvolvidos no Brasil, sete nos EUA e um na Austrália. Oito dessas pesquisas estão publicadas em inglês e oito em português. Quanto à instituição de origem dos autores, dez eram vinculados a universidades e um dos artigos era vinculado a uma Escola de Enfermagem dos EUA. No que tange ao ano de publicação, elas distribuíram-se entre os anos de 2012 e 2019.

Os resultados, organizados quanto à análise dos estudos primários, foram agrupados em duas categorias quanto à similaridade temática. Nos Quadros 1 e 2 destacam-se as características dos estudos primários incluídos na revisão, de acordo com a categoria delimitada. Com relação ao nível de evidência dos 11 estudos incluídos, apenas um teve evidência IV; os demais apresentaram evidência VI. Na sequência dos quadros será descrita uma síntese dos estudos primários selecionados, separadamente, de acordo com a categoria. Nesta síntese serão expostos os objetivos, local onde foi realizado o estudo, amostra investigada e principais resultados obtidos, a saber:

Categoria um: Assistência à saúde das gestantes encarceradas nas penitenciárias brasileiras $(n=5)$

O Quadro 1 apresenta as características dos estudos agrupados nesta categoria, destacando autor, ano, delineamento da pesquisa e nível de evidência. 
Quadro 1 - Características dos estudos primários agrupados na primeira categoria: assistência à saúde das gestantes encarceradas nas penitenciárias brasileiras

\begin{tabular}{|l|l|l|c|}
\hline Autor & Ano & \multicolumn{1}{|c|}{$\begin{array}{c}\text { Delineamento da } \\
\text { pesquisa }\end{array}$} & $\begin{array}{c}\text { Nivel de } \\
\text { evidência }\end{array}$ \\
\hline $\begin{array}{l}\text { E1 } \\
\text { Correa M }\end{array}$ & 2017 & Estudo qualitativo & VI \\
\hline E2 ${ }^{(13)}$ Galvão MCB, Davim RMB & 2013 & Estudo qualitativo & VI \\
\hline $\begin{array}{l}\text { E3 } \\
\text { Leite MF, Fúcoli RP, Santos JPS, Arazawa CY, Medeiros ICP, }\end{array}$ & 2014 & Estudo quantitativo & VI \\
\hline $\begin{array}{l}\text { E4 }{ }^{(15)} \text { Santos RMA, Linhares FMP, Morais SCRV, } \\
\text { Guedes TG, Guimarães ML }\end{array}$ & 2018 & Estudo quantitativo & VI \\
\hline $\begin{array}{l}\text { E5 } \\
\text { Sánchez AR, Larouzé B }\end{array}$ & 2016 & Estudo quantitativo & VI \\
\hline
\end{tabular}

Fonte: Elaboração própria.

Os cinco artigos primários vinculados a esta categoria estão descritos a seguir.

O estudo E1, realizado no Brasil, em unidades prisionais, contou com a participação de $22 \mathrm{MPL}$ gestantes, bem como mulheres cujos filhos encontravam-se com elas. Também participaram 19 funcionários que conviviam com as mães presas. Destacou-se que a existência de leis não garantiam sua efetivação, como foi comprovado nos relatos das MPL, que afirmaram violência durante o parto, pela equipe do hospital, desigualdade no tratamento, uso de algemas durante o parto e falta de acompanhante. A maior parte das mulheres entrevistadas não tinha iniciado o acompanhamento pré-natal quando foram presas ou estavam tendo atendimento de forma inapropriada. Elas ainda destacaram suas incertezas quanto ao parto, e demonstraram medo de não conseguirem ser levadas a tempo para a maternidade ${ }^{(12)}$.

No estudo E2, uma pesquisa descritiva qualitativa, desenvolvida no Complexo Penal Feminino Dr. João Chaves (CPFDJC) em Natal, Rio Grande do Norte, Brasil, tendo como participantes nove mulheres que vivenciaram a gravidez dentro do CPFDJC, analisou-se a deficiência da assistência à saúde e acompanhamento do pré-natal das gestantes encarceradas. Os resultados identificaram que a maioria das presidiárias não teve o acompanhamento do pré-natal durante a gestação, além da falta de assistência à saúde. Outros fatores relacionados com o desenvolvimento gestacional saudável também foram ressaltados no decorrer do estudo, como, por exemplo, a alimentação inadequada ${ }^{(13)}$.

No estudo E3, de corte transversal com aplicação de questionário, realizado em um presídio feminino em Campo Grande, Mato Grosso do Sul, Brasil, descreveram-se as características da assistência ao pré-natal e puerpério das mulheres detentas. O estudo foi realizado com 12 MPL, sendo 9 gestantes e 3 puérperas. A maioria das participantes relatou o uso de drogas ilícitas durante a gestação, sendo citadas a maconha e a pasta base, que podem causar complicações obstétricas e neonatais. A minoria das participantes relatou ter recebido orientações quanto aos cuidados com as mamas, aleitamento materno e pré-natal. A maioria das entrevistadas apontou que tinha adquirido algum tipo de Infecção Sexualmente Transmissível (IST), o que chama a atenção para a necessidade de ações de prevenção que precisam ser reforçadas durante a assistência ao pré-natal, de acordo com a realidade e a necessidade das detentas ${ }^{(14)}$.

O estudo E4, desenvolvido na Colônia Penal Feminina de Recife, Pernambuco, Brasil, com a participação de 18 mulheres que estavam amamentando durante o período da coleta de dados, identificou que os principais Diagnósticos de Enfermagem, de acordo com a taxonomia NANDA-1, são risco de vínculo mãe-filho prejudicado, padrão de sexualidade ineficaz e conhecimento deficiente sobre a amamentação. 
Apesar da existência de inúmeros protocolos e portarias que garantem a todos, estejam eles em privação de liberdade ou não, o direito aos serviços de saúde, os diagnósticos de enfermagem encontrados nesse grupo populacional demonstraram a vulnerabilidade à qual estão expostas, bem como a fragilidade dos serviços de saúde, que são ainda mais agravados devido ao ambiente no qual estão inseridas. A enfermagem exerce uma função importante nesse setor, ao levar informações às detentas, quebrando paradigmas e proporcionando uma melhora na qualidade de vida dessas mulheres privadas de liberdade ${ }^{(15)}$.

O estudo $\mathrm{E} 5$, realizado com 241 mães em unidades prisionais femininas das capitais e regiões metropolitanas do Brasil, descreveu que as mães eram majoritariamente jovens, com alta paridade e estavam grávidas quando foram presas.
Durante a gestação, a mulher tem o direito, preconizado pela Lei $\mathrm{n}^{\circ} 11.634 / 2007$, de ser vinculada à maternidade onde o parto acontecerá, com o objetivo de prepará-la para o momento, mas as MPL não se beneficiam desse direito. Destacou-se, nesse estudo, que a assistência ao pré-natal iniciou-se tardiamente e de forma inadequada em relação ao número de consultas, e um número importante de mulheres relatou ter sofrido violência na maternidade ${ }^{(16)}$.

Categoria dois: Assistência à saúde das gestantes encarceradas nas penitenciárias internacionais $(n=6)$

O Quadro 2 destaca características dos estudos agrupados nesta Categoria, segundo autor, ano, delineamento da pesquisa e nível de evidência.

Quadro 2 - Características dos estudos primários agrupados na segunda categoria: assistência à saúde das gestantes encarceradas

\begin{tabular}{|l|l|l|c|}
\hline Autor & Ano & \multicolumn{1}{|c|}{$\begin{array}{c}\text { Delineamento da } \\
\text { pesquisa }\end{array}$} & $\begin{array}{c}\text { Nivel de } \\
\text { evidência }\end{array}$ \\
\hline E6 ${ }^{(17)}$ Lepper LET, Trivedi S, Anakwe A & 2018 & Estudo quantitativo & VI \\
\hline E7 $^{(18)}$ Walker JR, Hilder L, Levy MH, Sullivan EA & 2014 & Estudo quantitativo & IV \\
\hline $\begin{array}{l}\text { E8 } \\
\text { Forestell C }\end{array}$ & 2017 & Estudo quantitativo & VI \\
\hline E9 ${ }^{(20)}$ Ferszt GG, Clarke JG & 2012 & Estudo quantitativo & VI \\
\hline $\begin{array}{l}\text { E10 } \\
\text { Byrnes Loshin LS, Sissoko DRG, Neumann G, Sufrin C, }\end{array}$ & 2019 & Estudo quantitativo & VI \\
\hline E11 & & & VI \\
\hline
\end{tabular}

Fonte: Elaboração própria.

A esta categoria foram vinculados seis artigos primários, como descrito a seguir.

No estudo E6, desenvolvido em um centro correcional do Centro-Oeste dos EUA, realizado por meio de um workshop com 25 gestantes encarceradas, com o objetivo de educá-las sobre os cuidados básicos e pessoais de seu período gestacional e cuidados com o bebê, foram abordados temas como: nutrição, exercício e saúde durante a gravidez; drogas, álcool e uso de tabaco; parto e pós-parto quando encarcerada; seus sentimentos sobre tornar-se mãe; como cuidar de seu bebê; e planejamento familiar.
Foram oito sessões de workshop, no geral com 90 minutos de duração, no qual, a cada sessão, as mulheres começavam completando um pré-teste sobre seu conhecimento prévio sobre o tópico a ser discutido na sessão e terminavam com a conclusão de um pós-teste, a fim de avaliar os conhecimentos obtidos no workshop. Os resultados foram insatisfatórios, pois a maioria delas não manifestou mudanças em seu conhecimento sobre os assuntos. Realizar a reestruturação das sessões de conhecimento, criar atividades que incentivem a interação entre o pessoal da prisão, os coordenadores do programa e as internas e 
adaptar o material para atender às necessidades individuais de cada grávida encarcerada, pode ser mais eficaz, criando, assim, uma atmosfera que incentive a aprendizagem ${ }^{(17)}$.

No estudo E7, realizado em New South Wales, na Austrália, foram utilizados dados de saúde coletados em uma unidade correcional, com o objetivo de determinar se as mulheres encarceradas, durante o período gestacional, obtiveram melhores resultados maternos e perinatais, em comparação com as mulheres da comunidade externa. Os resultados apontaram que o grupo de mulheres encarceradas era mais propenso a iniciar o pré-natal após a $20^{\mathrm{a}}$ semana de gestação do que o grupo de mulheres gestantes da comunidade externa. Apesar do acesso limitado aos serviços de saúde durante o período gestacional, as presas grávidas apresentaram menores taxas de morbidades maternas, como diabetes gestacional e hipertensão. Em relação ao tipo de parto, não houve diferença significativa do número de parto cesariana entre os dois grupos de mulheres ${ }^{(18)}$.

No estudo E8, a pesquisa teve como foco as práticas de assistência em saúde durante o período gestacional das mulheres encarceradas em 53 prisões nos EUA. Os resultados mostraram que $37,7 \%$ das prisões realizavam teste de gravidez no momento da admissão da mulher. Todas as cadeias relataram ter assistência médica no local, sendo 41,2\% internamente e 58,8\% contratados. Em 31,4\% das instalações, as presidiárias eram transportadas quando necessitavam de atendimento especializado em ginecologia e obstetrícia. Sobre a realização de ultrassom obstétrico, apenas $44 \%$ das prisões prestavam essa assistência. Durante o trabalho de parto, 17,4\% das instalações relataram fazer uso de algemas nas encarceradas ${ }^{(19)}$.

No estudo E9, a pesquisa teve como objetivo obter informações e avaliar as práticas assistenciais de saúde para as mulheres grávidas privadas de liberdade. Foram identificadas 50 prisões estaduais nos EUA, dentre as quais 32 concordaram em participar da pesquisa, porém, no estudo, constaram 19 prisões participantes. Em relação à assistência do pré-natal, 12 instalações relataram encaminhar a gestante para o serviço especializado de cuidados no pré-natal. Quanto à alimentação, a maioria das prisões refere oferecer uma dieta de baixo valor nutricional. Das 19 instituições que participaram da pesquisa, 2 unidades relataram usar cintos e correntes ao transportar as mulheres para o hospital, 8 usavam algum tipo de restrição durante o trabalho de parto e 6 a utilizavam no momento da entrega do recém-nascido para a mãe $e^{(20)}$.

No estudo E10, realizado nos EUA, 923 membros da Associação de Saúde da Mulher, Enfermeiras Obstétricas e Neonatais (AWHONN) foram convidados, entre julho e setembro de 2017, para responder um questionário on-line. A pesquisa teve como objetivo relatar a experiência de Enfermeiras Obstétricas na assistência às mulheres grávidas encarceradas durante gravidez, parto e pós-parto em determinado hospital. Dos participantes da pesquisa, 690 relataram experiência de cuidados perinatais com mulheres grávidas encarceradas, 67 relataram sentir-se inseguros. Sobre as justificativas para o uso de algemas, 347 informaram seguir o protocolo; 318, devido ao risco de fuga; 159 , pelo risco de danos a terceiros; e 64, para prevenir risco de dano para si mesmo. Apenas 22 participantes relataram ter conhecimento sobre leis quanto ao uso de algemas na assistência ao cuidado, no estado em que exerce ${ }^{(21)}$.

O estudo E11, realizado em uma unidade perinatal dos EUA com 35 enfermeiros, no qual apenas 11 responderam ao questionário da pesquisa, teve como objetivo conhecer a experiência desses profissionais na assistência às mulheres privadas de liberdade durante o parto e pós-parto. Os resultados mostraram que muitos enfermeiros relataram dificuldades em prestar uma boa assistência para a mulher durante o trabalho de parto, devido a alguns desafios enfrentados, a exemplo do uso de algemas e correntes, além da presença de guardas que acompanhavam as mulheres, o que causava incômodo e invadia sua privacidade. Outra questão dolorosa para os enfermeiros era a separação da mãe e seu filho logo após o parto, quando a mãe retornava para a prisão. Os resultados indicaram que 
uma parte dos enfermeiros entrevistados manifestou atitudes de julgamento com as pacientes encarceradas $^{(22)}$.

\section{Discussão}

A análise do perfil epidemiológico das mulheres brasileiras encarceradas evidencia uma população composta por mulheres jovens, de baixo nível socioeconômico e educacional, que possuíam uma iniciação precoce da vida sexual e alto índice de gestações ${ }^{(23)}$, além de mães solteiras e afrodescendentes ${ }^{(24)}$. Conhecer o perfil dessa população é importante para identificar as principais vulnerabilidades, para que as estratégias de promoção, proteção e recuperação da saúde sejam condizentes com a realidade vivenciada. As mulheres podem adentrar no cárcere em qualquer fase do ciclo reprodutivo ou gestacional, independentemente do seu contexto social. Segundo a Lei de Execuções Penais (LEP) n⿳o 7.210/1984 e a Lei no $11.942 / 2009$, é uma obrigação do estado prestar acompanhamento médico no pré-natal, parto e pós-parto, garantindo assistência de forma integral à saúde da mãe e do bebê, e também condições mínimas de assistência $^{(7)}$.

Nesta revisão, pôde-se analisar, na categoria um, de estudos nacionais, que há evidências quanto à falta de orientações acerca dos cuidados com as mamas, aleitamento materno e pré-natal $^{(14)}$, ausência de acompanhamento durante a gestação, além da falta de assistência à saúde ${ }^{(13)}$, mulheres que iniciaram tardiamente e de forma inadequada as consultas ou relataram ter sofrido violência na maternidade ${ }^{(16)}$. Estudos destacaram a violência perpetrada pela equipe do hospital durante o parto, desigualdade no tratamento, uso de algemas e falta de acompanhante $^{(12)}$. Embora não tenha sido identificado um estudo abrangente, com as diferentes penitenciárias do país, esta revisão conseguiu abranger penitenciárias do Centro-Oeste, Nordeste e Sudeste. Em todas demarcaram-se violações dos direitos garantidos às MPL.

Os estudos internacionais, por sua vez, apontaram dados mais abrangentes, como no estudo $^{(20)}$ que avaliou 19 penitenciárias dos EUA. A maioria delas relatou encaminhar a gestante para o serviço especializado de cuidados no pré-natal. Quanto à alimentação, a maioria das prisões referiu oferecer uma dieta de baixo valor nutricional, ofertando qualidade no acompanhamento da gestante. No entanto, quatro estudos ${ }^{(19-22)}$ citaram o uso de algemas durante o parto. Como justificativa tem-se questões de protocolos, risco de fuga e risco de danos a terceiros ou a si ${ }^{(21)}$.

Nesse contexto, o uso de algemas é algo comumente implantado dentro dos sistemas prisionais, não havendo exceção ou tratamento diferenciado para mulheres gestantes ou mães com filhos de colo ${ }^{(13)}$. No Brasil, destaca-se o Projeto de Lei do Senado $n^{\circ} 513 / 2013$, que altera as leis de execução penal e traz alterações para o uso de qualquer tipo de contenção durante o parto. Nessa perspectiva, um estudo nos EUA evidenciou a evolução de algumas leis estaduais, que adotaram um olhar mais humanizado para essas gestantes e puérperas, abolindo o uso de algemas durante o processo de transporte e/ou parto hospitalar ${ }^{(25)}$.

Outro problema identificado nos estudos nacionais relaciona-se à ausência de acompanhante durante o parto, como deveria ser garantido pela Lei $\mathrm{n}^{\circ}$ 11.108, de 7 de abril de 2005, que prevê, no art. 19: "O Sistema Único de Saúde - SUS, da rede própria ou conveniada, ficam obrigados a permitir a presença, junto à parturiente, de 1 (um) acompanhante durante todo o período de trabalho de parto, parto e pós-parto imediato"(25:1) Ressalta-se que essa situação independe do contexto de cárcere ou marginalização da mulher. No tocante à comunicação entre a penitenciária e os familiares, estudo ${ }^{(16)}$ identificou que era precária, pois cerca de $\%$ das famílias não foram avisadas quanto ao início do trabalho de parto das MPL.

No Brasil, em Minas Gerais, há o Centro de Referência à Gestante Privada de Liberdade, inaugurado em 2009, com o objetivo de permitir que as mães presas ficassem com seus filhos por mais tempo e promovesse um acompanhamento integralizado e adequado às mulheres grávidas ${ }^{(24)}$. 
A exemplo de Minas Gerais, os centros de referência podem ser estratégicos na perspectiva de atuação como guias operantes das leis que regem as penitenciárias femininas brasileiras, na garantia de que essas MPL possam ser assistidas integralmente, mesmo em meio ao caos que as ronda.

Uma reflexão é relevante acerca da organização e atuação da gerência das penitenciárias brasileiras no cuidado e atenção à saúde das detentas. Na França, por exemplo, o cuidado em saúde está além da administração da penitenciária e é garantido por lei, ficando sob a responsabilidade dos hospitais da região onde está localizada a penitenciária ${ }^{(16,26)}$. Logo, o acompanhamento da gestante durante seu pré-natal dar-se-á por meio da equipe do hospital e traria mais confiança às MPL e menos ansiedade ao processo de parto e pós-parto ${ }^{(16)}$. A humanização do atendimento, necessária em todos os ambientes de cuidado, ainda encontra entraves oriundos, muitas vezes, dos protocolos do sistema prisional.

Os profissionais de enfermagem estão intimamente ligados a essas MPL, como identificado em estudo $^{(22)}$ no qual as enfermeiras relataram dificuldades em prestar uma boa assistência durante o trabalho de parto, por estarem cuidando de MPL. No Brasil, o profissional de enfermagem é o principal responsável pela assistência à saúde dessas mulheres, porém, devido à falta de recursos humanos e materiais, além de dimensionamento irregular, tem-se sobrecarga do trabalho que compromete a qualidade do atendimento prestado ${ }^{(5,27)}$. A enfermagem brasileira está bem posicionada para exercer um cuidado com atenção integral ao qual essas MPL têm direito, porém é necessário oferecer subsídios mínimos e dignos para garantir a qualidade do serviço.

Os achados desta investigação limitam-se ao estudo de artigos disponíveis em três portais de busca. Entretanto, sabe-se que há outros portais que podem conter dados complementares a esta revisão, no entanto, como se tratam de bases de dados com acesso pago, e considerando que os pesquisadores não obtiveram recursos para o financiamento específico desse acesso, optaram pelas de acesso gratuito.

\section{Conclusão}

O desenvolvimento do presente estudo possibilitou analisar as produções científicas nacionais e internacionais a respeito do cuidado em saúde oferecido às mulheres privadas de liberdade durante o pré-natal, contribuindo para o desenvolvimento de uma reflexão crítica sobre a realidade vivenciada no contexto do cárcere. Foram evidenciadas falhas no cumprimento da legislação vigente, no que se refere aos diretos das gestantes no sistema prisional. Além disso, em alguns estados não existem políticas públicas que assegurem o acesso a uma assistência ao pré-natal qualificada, o que aponta para a necessidade de uma reformulação ou discussão das políticas públicas relacionadas ao sistema prisional e à capacitação dos profissionais da área da saúde para atender essa população.

A realização deste estudo sugere a necessidade de novas pesquisas que investiguem a assistência de enfermagem no contexto prisional, possibilitando refletir a prática de cuidado e ampliando as possibilidades de humanização do atendimento à mulher que vivencia a maternidade no cárcere. Constatou-se baixo número de estudos que abordassem a temática no Brasil. Os poucos que tratam do assunto reforçam as leis vigentes e a dificuldade de colocá-las em prática.

Conclui-se que a vivência do pré-natal no cárcere está repleta de dificuldades e peculiaridades, a depender da penitenciária, exigindo dos gestores e dos profissionais de saúde um olhar atento às necessidades da mulher, da criança e de seus familiares, na tentativa de minimizar os impactos do contexto prisional na qualidade de vida das pessoas envolvidas.

\section{Fontes de Financiamento:}

Artigo financiado pela Universidade Federal da Fronteira Sul sob registro PES-2019-0516. 


\section{Colaborações:}

1 - concepção, projeto, análise e interpretação dos dados: Ana Carolina Sales, Gabrielle Kimie Pinheiro Nakada e Rafaela Azevedo Abrantes de Oliveira Simoneti;

2 - redação do artigo e revisão crítica relevante do conteúdo intelectual: Ana Carolina Sales, Gabrielle Kimie Pinheiro Nakada, Mateus Rodrigo Palombit, Vander Monteiro Conceição, Sueli Santiago Baldan, Elaine Miguel Delvivo Farão e Rafaela Azevedo Abrantes de Oliveira Simoneti;

3 - aprovação final da versão a ser publicada: Vander Monteiro Conceição, Sueli Santiago Baldan, Elaine Miguel Delvivo Farão e Rafaela Azevedo Abrantes de Oliveira Simoneti.

\section{Referências}

1. World Prison Brief. World Female Imprisonment List (fourth edition) [Internet]. London; 2017 [cited 2019 Jun 15]. Available from: http://www.prisonstudies.org/news/worldfemale-imprisonment-list-fourth-edition

2. Brasil. Ministério da Justiça e Segurança Pública. Departamento Penitenciário Nacional. Infopen - Levantamento Nacional de Informações Penitenciárias [Internet]. Brasília; 2016 [cited 2019 Apr 3]. Available from: http://dados.mj.gov. br/dataset/infopen-levantamento-nacional-deinformacoes-penitenciarias

3. Brasil. Ministério da Justiça e Segurança Pública. Departamento Penitenciário Nacional. Infopen - Levantamento Nacional de Informações Penitenciárias [Internet]. Brasília; 2014 [cited 2019 Apr 3]. Available from: http://dados.mj.gov. $\mathrm{br} /$ dataset/infopen-levantamento-nacional-deinformacoes-penitenciarias

4. Scherer ZAP, Scherer EA, Santos MA, Souza J, Pillon SC, Scherer NP. Freedom-deprived women: social representations of prison, violence, and their consequences. Rev Bras Enferm. 2020;73(3):e20180781. DOI: http://dx.doi. org/10.1590/0034-7167-2018-0781

5. Graça BC, Mariano MM, Gusmão MAJX, Cabral JF, Nascimento VF, Gleriano JF, et al. Dificuldades das mulheres privadas de liberdade no acesso aos serviços de saúde. Rev Bras Promoç Saúde.
2018 abr-jun;31(2):1-9. DOI: http://dx.doi. org $/ 10.5020 / 18061230.2018 .7374$

6. Brasil. Ministério da Saúde. Secretaria de Atenção à Saúde. Departamento de Ações Programáticas Estratégicas. Plano Nacional de Saúde no Sistema Penitenciário [Internet]. Brasília; 2004 [cited 2019 Apr 3]. Available from: http://bvsms.saude.gov.br/ bvs/publicacoes/cartilha_pnssp.pdf

7. Souza GC, Cabral KDS, Leite-Salgueiro CDB. Reflexões sobre a assistência em enfermagem à mulher encarcerada: um estudo de revisão integrativa. Arq Ciênc Saúde UNIPAR. 2018 jan/abr;22(1):55-62. DOI: 10.255110/arqsaude. v22i1.2018.6240

8. Mendes KDS, Silveira RCCP, Galvão CM. Uso de gerenciador de referências bibliográficas na seleção dos estudos primários em revisão integrativa. Texto Contexto Enferm. 2019;28:e20170204. DOI: https:// dx.doi.org/10.1590/1980-265X-TCE-2017-0204

9. Souza MT, Silva MD, Carvalho R. Revisão integrativa: o que é e como fazer. Einstein (Impr.). 2010;8:102-6.

10. Mother D, Liberati A, Tetzlaff J, Altman DG. The PRISMA Group. Preferred Reporting Items for Sistematic Reviews and Meta-Analyses: The PRISMA Statement for reporting systematic reviews and meta-analyses of studies that evaluate health care interventions: explanation and elaboration. J Clin Epidemiol. 2009 Jul;62(10):1006-12. DOI: http://dx.doi.org/10.1136/ bmj.b2700

11. Melnyk BM, Fineout-Overholt E. Making the case for evidence-based practice. In: Melnyk BM, Fineout-Overholt E. Evidence-based practice in nursing \& healthcare. A guide to best practice. Philadelphia: Lippincot Williams \& Wilkins; 2005. p. 3-24.

12. Diuana V, Ventura M, Simas L, Larouzé B, Correa M. Direitos reprodutivos das mulheres no sistema penitenciário: tensões e desafios na transformação da realidade. Ciênc Saúde Coletiva, 2017;21(7):2041-50. DOI: https://doi. org/10.1590/1413-81232015217.21632015

13. Galvão MCB, Davim RMB. Ausência de assistência à gestante em situação de cárcere penitenciário. Cogitare Enferm. 2013 jul/set;18(3):452-9. DOI: http://dx.doi.org/10.5380/ce.v18i3.33554

14. Pícoli RP, Santos JPS, Arazawa CY, Medeiros ICP, Leite MF, Furtado N, et al. Gestação e puerpério no cárcere: estudo descritivo da atenção à saúde. Rev baiana saúde pública. 2014 jan/mar;38(1):67-82. 
DOI: $\quad$ https://doi.org/10.22278/2318-2660.2014. v38.n1.a413

15. Santos RMA, Linhares FMP, Morais SCRV, Guedes TG, Guimarães ML. Nursing Diagnoses of incarcerated mothers. Rev Esc Enferm USP. 2018;52:e03338. DOI: http://dx.doi.org/10.1590/ S1980-220X2017024503338

16. Leal MC, Ayres BVS, Esteves-Pereira AP, Sánchez AR, Larouzé B. Nascer na prisão: gestação e parto atrás das grades no Brasil. Ciênc Saúde Coletiva. 2016;21(7):2061-70. DOI: https:// doi.org/10.1590/1413-81232015217.02592016

17. Lepper LET, Trivedi S, Anakwe A. Effectiveness of a Prison-Based Healthy Pregnancy Curriculum Delivered to Pregnant Inmates: A Pilot Study. J Correct Health Care. 2018;24(3):243-52. DOI: $10.1177 / 1078345818782988$

18. Walker JR, Hilder L, Levy MH, Sullivan EA. Pregnancy, prison and perinatal outcomes in New South Wales, Australia: a retrospective cohort study using linked health data. BMC Pregnancy and Childbirth. 2014;14:214. DOI: 10.1186/1471-2393-14-214

19. Kelsey CM, Medel N, Mullins C, Dallaire D, Forestell C. An Examination of Care Practices of Pregnant Women Incarcerated in Jail Facilities in the United States. Matern Child Health J. 2017;21(6):1260-6. DOI 10.1007/s10995-016-2224-5

20. Ferszt GG, Clarke JG. Health care of pregnant women in U.S. state prisons. J Health Care Poor Underserved. 2012;23(2):557-69. DOI: 10.1353/ hpu.2012.0048

21. Goshin LS, Sissoko DRG, Neumann G, Sufrin C, Byrnes L. Perinatal Nurses' Experiences With and Knowledge of the Care of Incarcerated Women During Pregnancy and the Postpartum Period. J Obstet Gynecol Neonatal Nurs. 2019;48(1):27-36. DOI: https://doi.org/10.1016/j.jogn.2018.11.002
22. Zust BL, Busiahn L, Janisch K. Nurses' Experiences Caring for Incarcerated Patients in a Perinatal Unit. Issues Mental Health Nurs. 2013;34(1):25-9. DOI: 10.3109/01612840.2012.715234

23. Macedo FRM, Terra FS, Santos SVM, Miranda RPR, Santana APA, Pereira SA. Perfil socioeconômico e saúde ginecológica de presidiárias. SANARE Rev Políticas Públicas [Internet]. 2016 [cited 2019 Oct 27];15(2):30-36. Available from: https://sanare. emnuvens.com.br/sanare/article/view/1035

24. Lopes TC, Pinheiro P. Trajetórias de mulheres privadas de liberdade: práticas de cuidado no reconhecimento do direito à saúde no Centro de Referência de Gestantes de Minas Gerais. Physis Rev Saúde Coletiva. 2016;26(4):1193-212. DOI: https://doi.org/10.1590/s0103-73312016000400007

25. Brasil. Lei $\mathrm{n}^{\mathrm{O}}$ 11.108, de 7 de abril de 2005 . Altera a Lei $\mathrm{n}^{\circ}$ 8.080, de 19 de setembro de 1990, para garantir às parturientes o direito à presença de acompanhante durante o trabalho de parto, parto e pós-parto imediato, no âmbito do Sistema Único de Saúde - SUS. Diário Oficial da União. Brasília, 2005 abr 8; Seção 1, página 1.

26. Rocha AP. Naître et vivre auprès de sa mère incarcérée: situation paradoxale entre prison et hôpital. Spirale [Internet]. 2010 [cited 2019 Oct 27]; 2(54):61-8. Available from: https://www.cairn.info/ revue-spirale-2010-2-page-61.htm\#

27. Araujo ET, Melo JKN,Crizóstomo CDD, Parentes KF, Costa AP, Gomes SV. Assistência de enfermagem às mulheres em cárcere privado. Rev Interd [Internet]. 2017 [cited 2019 Oct 27];10(2):151-9. Available from: https://revistainterdisciplinar.uninovafapi. edu.br/index.php/revinter/article/view/1186

Recebido: 2 de abril de 2020

Aprovado: 11 de julho de 2020.

Publicado: 23 de novembro de 2020

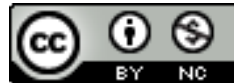

A Revista Baiana de Enfermagem utiliza a Licença Creative Commons - Atribuição-NãoComercial 4.0 Internacional.

https://creativecommons.org/licenses/by-nc/4.0/

Este artigo é de acesso aberto distribuído sob os termos da Licença Creative Commons (CC BY-NC).

Esta licença permite que outros remixem, adaptem e criem a partir do seu trabalho para fins não comerciais. Embora os novos trabalhos tenham de lhe atribuir o devido crédito e não possam ser usados para fins comerciais, os usuários não têm de licenciar esses trabalhos derivados sob os mesmos termos. 